\title{
Comparison of self-gripping mesh with mesh fixation with fibrin-glue in laparoscopic hernia repair (TAPP)
}

\author{
Cambal $\mathrm{M}^{1}$, Zonca $\mathrm{P}^{1,2}$, Hrbaty B \\ 1st Department of Surgery, Comenius University, Bratislava, Slovakia. mcambal@zoznam.sk
}

\begin{abstract}
Aim: Comparison of self-gripping mesh with fibrin-glue mesh fixation for laparoscopic hernia repair using TAPP technique.

Materials and methods: The trial has a prospective randomized design. The primary end-point was the evaluation of pain at 2 days, 1 month, and 3 months after surgery. The pain occurring 3 months after the surgery was considered as chronic pain.

Results: We have compared a group of 50 patients with self-gripping mesh with a group of 50 patients with fibrin glue mesh fixation using TAPP technique. There was no statistical difference between the basic group parameters (sex distribution, average age). There was no significant difference between the groups in terms of postoperative pain 1 month and 3 months after the surgery $(p>0.05)$. There was no patient with chronic pain at 3-month follow-up in our trial.

The mean operation time was 44 minutes in the group with self-gripping mesh and 48.5 minutes in thegroup with fibrin glue mesh fixation. There was a significant difference between both groups $(p=0.006)$

Conclusion: Both fixation methods appear to be a well-tolerated alternative to classical methods for mesh fixation with clips. According to our trial there is no difference in the postoperative pain incidence in selfgripping mesh and fibrin glue mesh fixation groups for laparoscopic hernia repair. Our data has showed that self-gripping mesh represents a tendency to a faster technique in comparison with fibrin-glue fixation. Both techniques are easy-to-use. There is no superior technique according to our trial (Tab. 2, Fig. 2, Ref. 23). Full Text in PDF www.elis.sk.

Key words: fibrin, self-gripping, mesh, groin, repair.
\end{abstract}

The surgery for inguinal hernia is one of the most frequently performed procedures in general surgery (1). Tension-free technique for groin repair is a standard technique of reparation nowadays. Both of the approaches - the open as well as laparoscopic one- use advantages of tension-free technique. A prosthetic mesh is required for tension-free reparation. It is believed that tensionfree technique is the best strategy to prevent recurrence of inguinal hernia (2). Laparoscopic methods such as trans-abdominal preperitoneal (TAPP) or totally extra-peritoneal (TEP) techniques offer postoperatively high comfort for patients. A disadvantage of the laparoscopic technique is the necessity of general anesthesia contrary to the open surgery which is possible to be performed under local anesthesia. It is important to provide proper fixation of prosthetic mesh to abdominal wall. The previously applied techniques with the usage of tissue-penetrating device could cause post-operative bleeding or chronic pain. Another often discussed factor producing postoperative pain is the mesh itself. Chronic pain influences negatively the quality of patient's life after the surgery. Chronic inguinal neuralgia is considered to be an important complication after hernia repair. Fibrin sealant or self anchoring

${ }^{1} 1$ st Department of Surgery, Komensky University, Bratislava, Slovakia, and ${ }^{2}$ Center for Visceral and Miniinvasive Surgery, Wesseling, Germany

Address for correspondence: M. Cambal, MD, 1st Department of Surgery, Comenius University, Mickiewiczova 13, SK. 81369 Bratislava, Slovakia. prosthesis were developed among others with the aim to decrease chronic pain. The usage of prosthesis without tissue penetrating device for fixation has become the most popular method in laparoscopic approach for hernia repair.

\section{Methods}

The aim of our trial is the comparison of self-gripping mesh with fibrin-glue mesh fixation for laparoscopic hernia repair with the usage of TAPP technique. The study was planned in accordance with the intention-to-treat principle. Our hypothesis supposed that the self-anchoring mesh is connected with a lower incidence of groin pain and a shorter operation time compared with the group with fibrin mesh fixation. The trial had a prospective randomized design. Two hospitals participated in the study. Informed consent was obtained from bypatients. The randomization into two arms was done before the surgery. The patients were randomized to TAPP with usage of either self-gripping mesh or fibrin glue mesh fixation. Numbered envelopes were used to randomize the patients into two groups. The study was approved by local ethics committee of both hospitals. The patients were informed about their participation in the trial. The aim was to include 100 patients into the trial. Patients with primary unilateral inguinal hernia eligible for TAPP were enrolled into trial. Men and women without age limitation were involved. Exclusion criteria were recurrent hernia 
Tab. 1. The local complications

\begin{tabular}{lcc}
\hline Local complications & Self-anchoring mesh & Fibrin glue mesh \\
\hline Hematoma & $3(6 \%)$ & $4(8 \%)$ \\
Seroma & $0(0 \%)$ & $0(0 \%)$ \\
Wound infection & $0(0 \%)$ & $0(0 \%)$ \\
Urinary retention & $2(4 \%)$ & $3(6 \%)$ \\
Local numbness & $2(4 \%)$ & $3(6 \%)$ \\
\hline
\end{tabular}

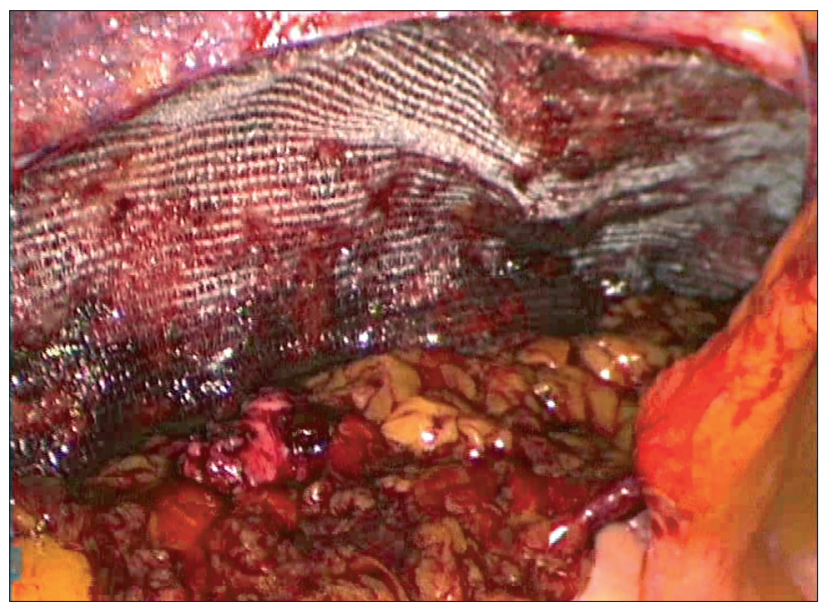

Fig. 1. Intraoperative picture of the self-gripping mesh in the left groin.

or huge scrotal hernia. Previous lower abdominal surgery with abdominal incisions below the umbilicus especially on the side of the hernia was another exclusion criterion. Patients who refused follow-up or patients who were considered unable to participate in the postoperative follow-up were also excluded. A high operative risk was a contraindication from an anesthetic point of view. Other exclusion criteria were intolerance to prosthesis, and general contraindication to laparoscopy.

The primary end-point was the evaluation of pain 2 days, 1 month, and 3 months after the surgery. The pain occurring 3 months after the surgery was considered as a chronic pain. Clinical examination was performed and pain was evaluated with the usage of Visual Analogue Scale (VAS). The secondary end-point was the evaluation of the operation time in both groups of patients using different fixation techniques. Data on local complication as local numbness, hematoma, seroma, wound infection, and urinary retention were collected. We focused also on the outcomes as a sensation of an extraneous body, and recurrence. Ease of product use was evaluated by three participating surgeons (Tab. 1).

All patients received a standard preparation including antibiotic prophylaxis and thrombosis prophylaxis according to relevant guidelines. A standard TAPP technique under general anesthesia was performed with the usage of $10 \times 15 \mathrm{~cm}$ mesh (Fig. 1).
Every patient was instructed to resume normal daily activities. Physically demanding activities and sports were recommended two weeks after the surgery.

An independent surgeon performed follow-up with a clinical examination 2 days, 1 month, and 3 months after the surgery. At the clinical examination the surgeon evaluated wound healing, event. hernia recurrence. The patients were asked whether they had any complaints especially regarding groin pain or another sensation. The patients were divided into three groups according to their postoperative pain complaints. The first group was without any complaints. The second group was with occasional discomfort or pain not interfering with daily activities. The third group experienced discomfort or pain interfering with daily activities (Tab. 2).

The following source materials were used for obtaining the data: operation chart, anesthetist chart, indication to operation, and the data from central data hospital system. The statistical evaluation of the difference between both groups of patients was performed using Student t-test. We used the statistical software SPSS version 15 for statistical analysis (SPSS Inc., Chicago, USA).

\section{Results}

We compared a group of 50 patients with self-gripping mesh with a group of 50 patients with fibrin-glue mesh fixation using TAPP technique in the period from February 2010 till June 2010. The enrollment of patients is showed in the study flow chart (Fig. 2).

There were 41 men and 9 women in the group with self-gripping mesh and 37 men and 13 women in the group with fibrin glue mesh fixation. The average age in the first and second group was $52.6 \pm 14.9$, and $50.3 \pm 15.8$ years, respectively $(\mathrm{p}=0.229)$. The distribution between men and women was proportional in both of the groups. There was no statistical difference between the basic groups' parameters.

There was no difference between complications ' incidence in both arms of the study.

Three-month follow-up was achieved in $94 \%$ of patients in the self-anchoring group and in $98 \%$ of patients in the group with fibrin-glue. There was no hernia recurrence during the follow-up of both the groups of patients at 3 months after the surgery. A sensation of an extraneous body was not observed as well.

The evaluation of pain in the group with self-gripping mesh follows: up until 2 days after the surgery 21 patients experienced occasional discomfort or pain and 24 patients discomfort or pain. Up until 1 month after the surgery 3 patients experienced occasional discomfort or pain and 1 patient discomfort or pain. The evaluation of pain results in the second group with mesh with fibrin fixation follows: up until 2 days after the surgery 14 patients experienced occasional discomfort or pain and 33 patients discomfort

\section{Tab. 2. Incidence of pain}

\begin{tabular}{llllll}
\hline & \multicolumn{2}{l}{ Self-gripping mesh } & & \multicolumn{2}{l}{ Fibrin glue fixation } \\
\hline & 2 days & 1 month & 3 months & 2 days & 1 month \\
No pain & $5 / 50$ & $44 / 48$ & $47 / 47$ & $3 / 50$ & $46 / 49$ \\
Occasional discomfort or pain & $21 / 50$ & $3 / 48$ & $0 / 47$ & $14 / 50$ & $1 / 49$ \\
Discomfort or pain & $24 / 50$ & $1 / 48$ & $0 / 47$ & $33 / 50$ & 0 \\
\hline
\end{tabular}




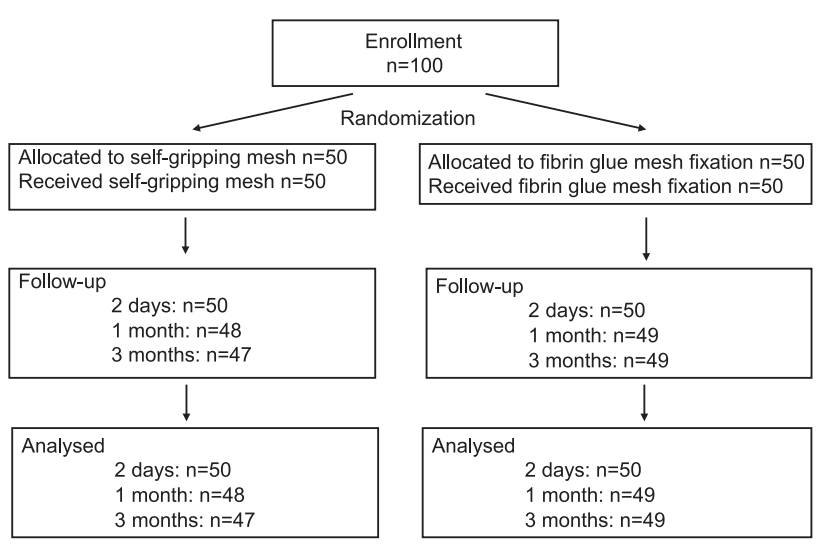

Fig. 2. Study flow chart.

or pain. Up until 1 month after the surgery 1 patient experienced occasional discomfort or pain and 2 patients discomfort or pain in the group with fibrin glues mesh. Four patients in the group with self-anchoring mesh experienced pain up until the one-month follow-up after the surgery; however, 3 months after the surgery they were free of pain. All data are summarized in table 2. Average evaluation of pain one month after the surgery in this group of patients was 3.2 according to VAS. Three patients in the group with fibrin glue mesh fixation experienced pain up until one-month after the surgery. One of them developed wound granuloma on the port side. Excision of the granuloma under local anesthesia was done and the patient experienced relief from pain. An average evaluation of pain one month after the surgery was 2.8 according to VAS in this group of patients. All patients improved after a course of oral non-steroidal anti-inflammatory analgesics. Nerve blocks were not necessary. There was no significant difference between the groups in terms of postoperative pain 1 month, and 3 months after the surgery $(p>0.05)$. There was no patient with chronic pain at 3-month follow-up in our trial.

The mean operation time was 44 minutes in the group with self-anchoring mesh and 48.5 minutes in the group with fibringlue mesh fixation. There was a significant difference between both groups $(\mathrm{p}=0.006)$.

Ease of product use was evaluated by three participating surgeons. They evaluated the group with self-anchoring mesh as very good. It was necessary to get used to "self-sticking" of the mesh with anchors, but learning curve did not take them much time. The positioning of self-anchoring mesh was short and this type of fixation allowed them to save time in contrast to the fibrin-glue group.

\section{Discussion}

The short-term outcome after TAPP hernia repair in both arms of our trial is very favorable. The recurrence of inguinal hernia after the operation is a main parameter in majority of the studies. Most of the recurrences occur within three months after the surgery and are attributable to technical error. No recurrence was observed in three-month follow-up in our study.
Inguinal hernia repair is the most common operation in general surgery. Chronic pain represents a serious problem after hernia repair, which was the focus of our trial. The reported incidence of chronic pain differs in a wide range according to literature. Some authors presented an incidence of chronic pain about $30 \%$ (3). Nienhuijs reported that the incidence of chronic pain varies between 0 and $75 \%$ after open mesh repair and between 0 and 29 $\%$ after laparoscopic repair (4). In a Danish questionnaire study 1652 patients undergoing surgery for inguinal or femoral hernia repair were included. The incidence $11-17 \%$ of chronic pain leading to various types of social disability was documented (5). Other authors have reported the necessity to referrar the patient to a pain clinic, $0.4 \%$ of patients after laparoscopic and $1-15 \%$ after open repair $(6,7)$. Kathouda compared the results of chronic pain when using laparoscopic and open technique with mesh. Metaanalysis of randomized controlled trials concluded that persistent pain occurred less frequently after laparoscopic repair than after open mesh placement (8). Other authors have also supported these results $(9,10)$.

It is important to pay special attention to the problem of chronic pain. The groin is innervated through the following nerves: nervi intercostales (Th VII-XII), nervus iliohypogastricus, nervus ilionguinalis, nervus genitofemoralis (ramus genitalis et ramus femoralis), and nervus cutaneus femoris lateralis. The injury of any of these nerves could cause neuralgia and other symptomatology. When using TAPP technique, as it was done in our trial, $\mathrm{n}$. genitofemoralis and less n. ilioinguinalis and $\mathrm{n}$. cutaneus femoris lateralis are injured mostly. The injury caused during the operation could lead to neuralgia after the surgery. However, the compression of nerves with the used material (stitches, clips, mesh) could be a reason for postoperative pain as well (11). The onset of groin pain could come with delay. A reason for complications could be the roll up of mesh, periostal reaction or development of cicatricial tissue in the surrounding of mesh with the following compression of nerves or development of perineural fibrosis

Mesh is usually fixed to the peritoneum by clips. The use of fibrin glue to fix the prosthesis to the parietal wall was introduced with the aim of elimination of postoperative pain, due to peritoneal stretching and nerve compression. Fibrin glue fixation began to be used in hernia repair from 2002 according to literature and quickly became popular as an alternative means of mesh fixation (12). Fibrin glue has many fields of application in medicine (13). Another possibility is fixation of mesh with cyanoacrylate. Cyanoacrylate has been used in surgery for 50 years (14). Recently, self-anchoring mesh without the necessity of usage of any other additional fixation has been introduced to the market. Self-gripping mesh has micro hooks on the surface to ensure its anchorage in the tissue. Additional fixation like fibrin glue or clips is not required. An interesting study in an animal setting about the strength of mesh incorporation was presented by Hollinky. He compared selfgripping mesh with hernia stapler (HS) and with fibrin glue (FG) and mesh without fixation (UM) in a rat model. After 5 days, the strength of incorporation was substantially higher for self-gripping mesh $\left(3.2 \mathrm{~N} / \mathrm{cm}^{2}\right)$ and hernia stapler $\left(2.7 \mathrm{~N} / \mathrm{cm}^{2}\right)$ compared with fibrin glue $\left(0.9 \mathrm{~N} / \mathrm{cm}^{2}\right)$ or mesh without fixation $\left(1.5 \mathrm{~N} / \mathrm{cm}^{2}\right)$. Af- 
ter two months, self-gripping mesh had a much greater strength of incorporation $\left(14.8 \mathrm{~N} / \mathrm{cm}^{2}\right)$ compared with all the other groups (HS $11.7 \mathrm{~N} / \mathrm{cm}^{2}$; FG $11.4 \mathrm{~N} / \mathrm{cm}^{2}$; UM $8.7 \mathrm{~N} / \mathrm{cm}^{2}$ ) (15).

Another reason for postoperative pain could be the type of mesh. A modern material has been developed as a high biocompatible material with minimal reaction of tissue with subsequent problems. Many types of light meshes are available (e.g. Vypro II, Premilene, Parietene light, TiMesh and others). At present the usage of these meshes has become a standard. The usage of lightweight meshes in inguinal hernia repair has beneficial effects on the quality of life. Some authors reported that the nerves could be damaged by suture entrapment or by contact with mesh. The probability of acute or chronic pain after surgery in these cases is very high (16). Schopf presented a study with titanium covered polypropylene mesh. Chronic pain was not very common in patients in his study. The reduction of the material load from 35 to $16 \mathrm{~g} / \mathrm{m}^{2}$ had further improved the clinical outcome by reducing chronic pain to a rare event. He pointed out that the role of staples in causing chronic pain following inguinal hernia repair may be overestimated and that the material of the mesh seems to be more important (17). The search with key words fibrin and hernia repair in PubMed showed that 90 trials have been published. The search for self-gripping mesh and hernia repair revealed only two studies. One of these studies was the Hollinsky study and another study by Chastan (18). Direct comparison of fibrin glue and self-gripping mesh has not been performed in a clinical setting according to PubMed search yet.

Chronic pain is defined as a pain or discomfort that lasts for more than 3 months after surgery according to its classification (19). The weakness of our study in the view of this definition is the length of the follow-up. At three-month follow-up we did not identify any patients with pain. The three-month follow-up did not make it possible to reveal a correct number of patients suffering from chronic pain, because of the possible delayed onset of pain. On the other side, this short follow-up allows a precise examination of patients with a low drop-out.

The quantification of pain is another problem. In our trial we used the Visual Analogue Scale (VAS) for subjective assessment of pain. VAS is a simple robust pain measurement tool. It can be used to measure severity as well as improvement. The VAS is usually designed as a $10 \mathrm{~cm}$ line with descriptors at each end. The $10 \mathrm{~cm}$ line allows for an easy measurement. Lord Kelvin (1824-1907) made an apt comment about this scale: "When you can measure it you are speaking about, and express it in numbers, you know something about it; but when you cannot measure it, your knowledge is of a meager unsatisfactory kind: It may be the start of knowledge, but you have scarcely in your thoughts, advanced to the stage of science. "In our trial the average evaluation of pain one month after the surgery in the group with self gripping mesh and in the group with fibrin glue was 3.2 and 2.8, respectively, according to VAS. The results in both groups are comparable without differences. At three-month follow-up we didn't perform this evaluation, because no patient with chronic pain was identified.

An interesting focus would be the analysis of risk factors for chronic pain in multivariable analysis, but we have not identified the patients experiencing chronic pain. It is possible that chronic pain occurs later, what might be identified at a planned one-year follow-up. The focus of interest will be the evaluation of patients ' parameters as patients' age, preoperative physical restriction, postoperative physical impairment, surgeon's age, and the time of recovery - similarly as it was performed in the study by Eklund (mentioned above). Eklund's study confirmed that patient's age, preoperative physical restriction, postoperative physical impairment, surgeon's age, and the time of recovery were risk factors.

The incidence of chronic pain depends on many factors. Our results were achieved because of the safe operation technique, and the usage of modern mesh material without tuckers. On the other side, our follow-up is only short-term. It is necessary to continue the follow-up of our patients and make evaluation one year after surgery. Chronic pain has been addressed prospectively by using standard definitions and allowing assessment of the degree of pain. Early postoperative complications were comparable to other studies $(20,21)$. We have not had a patient with the necessity to referr to pain management, or the necessity of a secondary procedure for pain control excluding the patient with granuloma. It was necessary to use only pain medication ad hoc in our setting. The treatment of chronic pain has more options. There is no high-level evidence-based treatment regime. Patients with chronic pain usually receive a combination of pain medication, local nerve blocks or an occasional surgical neurectomy. Loss presented a controlled trial to identify the optimal treatment modality. Patients received either repetitive nerve blocks with lidocain, corticosteroids and hyaluronic acid, or a 'tailored' surgical neurectomy. The initial results will be available by the end of 2010 (22). Neurectomy alone or in combination with mesh removal will be probably the proper option in patients with serious difficulties with chronic pain (23).

\section{Conclusion}

Both fixation methods appear to be a well-tolerated alternative to classical methods for mesh fixation with clips. According to our trial there is no difference in postoperative pain incidence in self-gripping mesh and fibrin glue mesh fixation groups for laparoscopic hernia repair. Our data show that self-gripping mesh represents a tendency to a faster technique compared with fibrin glue fixation. Both techniques are easy-to-use according to the attending surgeons. There is no superior technique according to our trial. The further development of biocompatible materials for tension-free groin repair is expected. A safe operation technique is the priority for the prevention of chronic pain after hernia repair. One-year or longer follow-up could reveal more facts. However, further investigation is needed to be done in more patients.

\section{References}

1. Rutkow IM, Robbins AW. Demographic, classificatory, and socioeconomic aspects of hernia repair in the United States. Surg Clin North Am 1993; 73: 413-426.

2. McCormack K, Scott NW, Go PM, Ross S, Grant AM; EU Hernia Trialists Collaboration (2003) Laparoscopic techniques versus open techniques for inguinal hernia repair. Cochrane Database Syst Rev 1: CD001785. 
3. Poobalan AS, Bruce J, King PM. Chronic pain and quality of life following open inguinal hernia repair. Br J Surg 2001; 88: 112-126.

4. Nienhuijs S, Staal E, Strobbe L, Rosman C, Groenewoud H, Bleichrodt R. Chronic pain after mesh repair of inguinal hernia: a systematic review. Am J Surg 2007; 194: 394-400.

5. Bay-Nielsen M, Perkins FM, Kehlet H. Pain and functional impairment 1 year after inguinal herniorrhaphy: a nationwide questionnaire study. Ann Surg 2001; 233: 1-7.

6. Hindmarsh AC, Cheong E, Lewis MP, Rhodes M. Attendance at a pain clinic with severe chronic pain after open and laparoscopic inguinal hernia repairs. Br J Surg 2003; 90: 1152-1154.

7. Courtney CA, Duffy K, Serpell MG, O'Dwyer PJ. Outcome of patients with severe chronic pain following repair of groin hernia. Br J Surg 2002; 89: 1310-1314.

8. Katkhouda N. A new technique for laparoscopic hernia repair using fibrin sealant. Surg Technol Int 2004; 12: 120-126.

9. Eklund A, Montgomery L, Bergkvist A, Rudberg C. Chronic pain 5 years after randomized comparison of laparoscopic and Lichtenstein inguinal hernia repair. Brit J Surg 2010; 97: 600-608.

10. Testini M, Lissidini G, Poli E, Gurrado A, Lardo D, Piccinni P. A single-surgeon randomized trial comparing sutures, $N$-butyl-2-cyanoacrylate and human fibrin glue for mesh fixation during primary inguinal hernia repair. Can J Surg 2010; 53 (3): 155-160.

11. Ninger V, Bis D. Pain following laparoscopic inguinal hernioplastics using the TAPP technique. Rozhl Chir 2006; 85 (7): 333-337.

12. Descottes B, Bagot d'Arc M. Fibrin sealant in inguinal hernioplasty: an observational multicentre study in 1,201 patients. Hernia 2009; 13 (5): 505-510.

13. Jourdan IC, Bailey ME. Initial experience with the use of N-butyl 2-cyanoacrylate glue for the fixation of polypropylene mesh in laparoscopic hernia repair. Surg Laparosc Endosc 1998; 8: 291-293.
14. Helbling C, Schlumpf R. Sutureless Lichtenstein: first results of a prospective randomised clinical trial. Hernia 2003; 7: 80-4.

15. Hollinsky C, Kolbe T, Walter I, Joachim A, Sandberg S, Koch T, Rülicke T. Comparison of a new self-gripping mesh with other fixation methods for laparoscopic hernia repair in a rat model. J Am Coll Surg 2009; 208 (6): 1107-1114.

16. Parviz, K., Amid, M. D. Surgical treatment for Postherniorrhaphy Neuropathic Inguinodynia: Triple Neurectomy with Proximal End Implantation. Contemp Surg 2003; 59: 276-280.

17. Schopf S, von Ahnen T, von Ahnen M, Schardey H. Chronic Pain after Laparoscopic Transabdominal Preperitoneal Hernia Repair: A Randomized Comparison of Light and Extralight Titanized Polypropylene Mesh. World J Surg 2010; 20.

18. Chastan P. Tension-free open hernia repair using an innovative selfgripping semi-resorbable mesh. Hernia 2009; 13 (2): 137-142.

19. Merskey HBN. Classification of Chronic Pain, Descriptors of Chronic Pain Syndromes and Definitions of Pain Terms ( $2^{\text {nd }}$ edn). IASP Press: Seattle, 1994.

20. Vanclooster P, Smet B, de Gheldere C, Segers L. Laparoscopic inguinal hernia repair: review of six years' experience. Acta Chir Belg 2001; 101: $135-138$

21. Masukawa K, Wilson SE. Is postoperative chronic pain syndrome higher with mesh repair of inguinal hernia? Am Surg 2010; 76 (10): $1115-1118$.

22. Loos MJ, Verhagen T, Scheltinga MR, Roumen RM A randomised controlled trial of injection therapy versus neurectomy for post-herniorrhaphy inguinal neuralgia: rationale and study design. Hernia 2010; 14 (6): 593-597.

23. Aasvang E, Kehlet H. Surgical management of chronic pain after inguinal hernia repair. Br J Surg 2005; 92: 795-801.

Received May 13, 2011. Accepted December 18, 2011. 